Classification

Physics Abstracts

86.30

\title{
Analyse des possibilités de fonctionnement en régime de désexci- tation des moteurs à aimants permanents
}

\author{
Bernard Multon, Jean Lucidarme et Laurent Prévond \\ LÉSIR, École Normale Supérieure de Cachan, URA CNRS D1375, 61 Avenue du Pdt Wilson, \\ 94235 Cachan Cedex, France
}

(Reçu le 30 novembre 1994, accepté le 30 janvier 1995)

\begin{abstract}
Résumé. - Cet article traite de l'extension de la plage de vitesse des moteurs (ou alternateurs) à excitation par aimants permanents et alimentés par convertisseur électronique. La tension et le courant sont limités en amplitude par l'alimentation électronique. L'objectif est d'obtenir une puissance proche de celle correspondant au régime de base sur une plage de vitesse étendue. Ceci nécessite une réduction de flux d'entrefer ou "désexitation" au delà de la vitesse de base. Une analyse paramétrique des caractéristiques du moteur est effectuée pour mettre en évidence l'influence de la réaction d'induit et de la saillance magnétique. Nous montrons qu'il existe une condition idéale pour obtenir une plage de fonctionnement à puissance constante théoriquement illimitée. La saillance magnétique permet d'accroître le facteur de puissance surtout lorsque $L_{\mathrm{d}}>L_{\mathrm{q}}$. Les principales hypothèses de cette étude sont l'absence de saturation, des f.e.m. et des courants sinusoïdaux et une induction spatiale sinusoïdale dans l'entrefer. Enfin, nous effectuons un bilan des structures de rotors permettant un fonctionnement en régime de désexcitation.
\end{abstract}

\begin{abstract}
In this paper, we study the extending of speed range of motors (or generators) with permanent magnet inductor and supplied by electronic converter. The amplitude of phase voltage and current waveforms are limited by electronic supply. The aim of this study is to achieve a maximum power near of the base speed one on an extended speed range. This require an airgap flux weakening so called "flux-weakening" above base speed. A parametric analysis of motor electromagnetic characteristics is made to show the influence of armature reaction and magnetic saliency on speed range. We show that exists an ideal condition to obtain a constant power speed range theoretically unlimited. Magnetic saliency permits to enhance the power factor especially when $L_{\mathbf{d}}>L_{\mathbf{q}}$. As main hypotheses, we consider no saturation, e.m.f. and current sine waveforms and a sinusoidal airgap flux density. Finally, we recapitulate the permanent magnet rotor structures able to obtain a flux-weakening operation.
\end{abstract}

\section{Introduction}

Nous nous intéressons ici aux limites de fonctionnement, dans le plan couple-vitesse, dues, d'une part, à la tension continue d'alimentation de l'onduleur et à son courant maximal commutable et, d'autre part, aux caractéristiques électromagnétiques de la machine (flux produit 
par les aimants, inductances dans les axes direct et transverse). L'objectif principal est d'analyser le phénomène de "désexcitation" (Flux-Weakening) des moteurs à aimants permanents à la lumière des divers travaux effectués sur le sujet $[3,4,6,10,21,23]$ et de faire un bilan des structures d'inducteurs adaptées à ce type de fonctionnement. Rappelons que le fonctionnement en régime de désexcitation ("fonctionnement à puissance constante") est recherché dans un certain nombre d'applications comme la traction électrique (routière ou ferroviaire), les entraînements de broches de machines-outils, ceux de gouvernes d'avions... Ce mode de fonctionnement est aussi particulièrement intéressant dans les générateurs à vitesse variable (alternateurs d'automobiles, avions, éoliennes...). Dans cet article, l'analyse sera effectuée en moteur mais les conclusions sont parfaitement valables en fonctionnement générateur.

Les principales hypothèses sont les suivantes : circuit magnétique non saturé, force électromotrice sinusoïdale, alimentation en courants sinusoïdaux, induction d'entrefer spatiale sinusoïdale (décomposition en deux axes $d$ et $q$ ), résistance d'induit négligée (hypothèse valable pour les moteurs dont le couple est supérieur au $\mathrm{Nm}$ environ et fonctionnant à des vitesses de quelques $1000 \mathrm{tr} / \mathrm{mn})$.

\section{Puissance apparente silicium. Facteur de dimensionnement}

Afin de quantifier la puissance installée en semi-conducteurs et, éventuellement, d'effectuer des comparaisons entre systèmes d'entraînements électriques, nous avons, pour le régime de base (couple maximal ou nominal et vitesse de base), défini [9] les notions :

- de "puissance apparente silicium" (V.A Si) :

$$
P_{\mathrm{Si}}=n \cdot U_{\mathrm{Max}} I_{\mathrm{Max}}
$$

où $n$ est le nombre total de semi-conducteurs commandés et où $U_{\operatorname{Max}}$ et $I_{\text {Max }}$ sont leurs contraintes maximales en tension et courant;

- et de facteur de dimensionnement :

$$
f_{\mathrm{d}}=\frac{P_{\mathrm{Si}}}{P}
$$

où $P$ est la puissance mécanique utile définie par :

$$
P=\eta \cdot P_{\mathrm{e}}
$$

$\eta$ est le rendement du moteur et $P_{\mathrm{e}}$ est la puissance électrique absorbée par le moteur.

Même si cette notion est insuffisamment précise, il faudrait en effet tenir compte des pertes de conduction (combinaison courants efficace et moyen) et de commutation, elle permet une première quantification utile.

Par exemple, le hacheur série (Fig. 1) alimentant un moteur à collecteur conduit à la valeur minimale de $f_{\mathrm{d}}$ égale à $1 / \eta$ (dans le cas idéal où les ondulations de tension d'alimentation et du courant moteur sont négligeables). Un hacheur 4 quadrants donnerait :

$$
f_{\mathrm{d}}=\frac{4}{\eta}
$$

L'onduleur triphasé qui nous intéresse dans le cas des moteurs à aimants (Fig. 1), avec modulation sinusoïdale du courant conduit à :

$$
f_{\mathrm{d}}=\frac{8}{\eta \cdot \cos \varphi}
$$



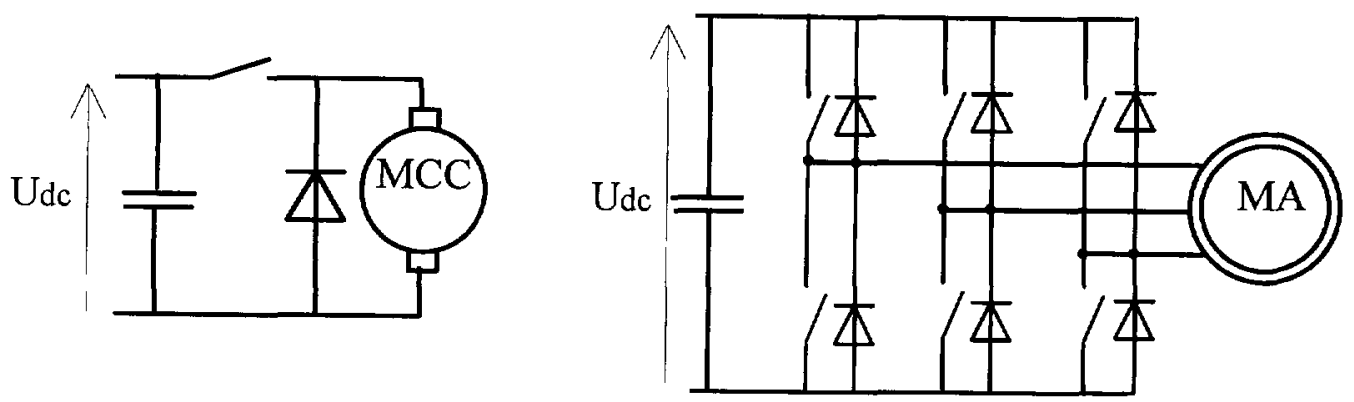

Fig. 1. - Structures de convertisseurs pour moteurs à courant continu et moteurs triphasés à courant alternatif.

[Electronic converter structures for DC motors and for three phase AC motors.]

où $\varphi$ est l'angle de déphasage du courant par rapport au fondamental de la tension. Il faut ainsi plus de 8 fois la "quantité" de silicium d'un hacheur série répartie sur 6 composants... Notons que pour un onduleur diphasé à deux ponts complets ( 8 transistors), le facteur de dimensionnement a la même expression.

Si $U_{\mathrm{dc}}$ est la tension continue d'alimentation de l'onduleur triphasé, l'amplitude maximale de la tension simple fondamentale vaut, si le rapport cyclique maximal de découpage tend vers 1 :

$$
V_{1 \mathrm{M}}=\frac{U_{\mathrm{dc}}}{2}
$$

Si l'on considère la transformation classique, triphasée-diphasée, l'amplitude maximale de la tension fondamentale dans les axes $d$ et $q$ vaut :

$$
V_{\mathrm{d}, \mathrm{q} 1 \mathrm{M}}=\sqrt{\frac{3}{2}} \cdot \frac{U_{\mathrm{dc}}}{2}=0,61 \cdot U_{\mathrm{dc}}
$$

Notons qu'une commande de type trapèze (forces électromotrices trapézoïdales et courants en quasi-créneaux) conduit dans le cas où le moteur est faiblement inductif (petites dimensions) à une valeur plus faible de $f_{\mathrm{d}}$ :

$$
f_{\mathrm{d}}=\frac{6}{\eta}
$$

Si l'on prend en compte l'inductance du bobinage, $f_{\mathrm{d}}$ se dégrade et on peut le réduire en effectuant une avance de l'angle de mise en conduction (on arrive d'ailleurs à une situation où les trois phases, au lieu de deux, sont alimentées en même temps) [15].

En pratique, la tension maximale d'alimentation est limitée par la source continue et le courant crête doit rester inférieur à une valeur maximale. Cette dernière correspond généralement au couple à basse vitesse. Pour maintenir le courant lorsque la vitesse augmente, la f.e.m. ainsi que la tension de réaction d'induit croissent, à partir d'une vitesse dite vitesse de base, la tension maximale disponible est juste égale à la somme vectorielle des tensions moteur. En général, c'est à cette vitesse que l'on a atteint la puissance maximale du moteur et c'est pour ce point de fonctionnement que la puissance apparente silicium est définie. Au delà de cette vitesse, si l'on veut convertir une puissance la plus élevée possible (voire constante), il est nécessaire de "désexciter" (ou "défluxer") le moteur pour continuer à injecter un courant suffisant. 
Le nombre de spires du bobinage (pour une phase) est généralement déterminé pour le fonctionnement à la vitesse de base et au couple "nominal", sa valeur est sensiblement proportionnelle à la tension d'alimentation. Notons que le facteur de dimensionnement, la puissance silicium et le facteur de puissance ne dépendent pas de la tension continue et du nombre de spire ainsi choisis et définis.

Dans les moteurs à excitation bobinée, cette désexcitation est possible par action directe sur le flux inducteur. Mais dans le cas des moteurs à aimants permanents, elle semble moins évidente et n'est, en réalité, possible que grâce à une orientation adéquate du champ de réaction d'induit. On parle alors de "désexcitation", "défluxage", ou encore de réduction de champ.

\section{3. Équations de la macine synchrone à aimants permanents - Désexcitation}

3.1. MaChine SANS SAILlanCE. - Pour commencer l'analyse simplement, avec un minimum de paramètres, considérons le cas de la machine synchrone à aimants sans saillance magnétique ou "à pôles lisses". Nous appelons saillance magnétique la différence de réluctance du trajet du flux induit dans les axes direct et transverse à l'axe du flux inducteur. Les hypothèses principales sont les suivantes : absence de saturation magnétique, forces électromotrices sinusoïdales et courants sinusoïdaux (régulation idéale). Notons que dans la référence [30], l'auteur a montré que la saturation modifiait très peu les caractéristiques dans la zone de fonctionnement dite "à puissance constante", essentiellement parce que le flux est justement réduit.

Dans la présente étude, on ne prend pas en compte la résistance des bobinages d'induit, cette hypothèse peut être justifiée par le fait que l'on ne ne considère que les hautes vitesses (alimentation sous la tension maximale, donc chute $R . i$ faible devant $v$ ) et le domaine de puissances relatif aux applications de désexcitation (traction, broches).

Dans ces conditions, le couple moyen s'exprime par :

$$
T=3 \cdot \phi_{\mathrm{f}} \cdot I_{\mathrm{q}} \quad \text { où } \quad I_{\mathbf{q}}=I \cdot \cos \psi \quad \text { et } \quad I_{\mathrm{d}}=I \cdot \sin \psi
$$

$I_{\mathrm{q}}$ et $I_{\mathrm{d}}$ sont les composantes transverse et directe (correspondant aux composantes de la f.m.m. dans l'axe du flux inducteur donc magnétisante ou démagnétisante) du courant d'une phase, $\phi_{\mathrm{f}}$ est la valeur efficace du flux inducteur (sinusoïdal) d'une phase, $\psi$ est l'angle d'autopilotage, sur le diagramme vectoriel de la figure 2 : angle de $\mathbf{I}$ vers $\mathbf{E}$ (la f.em.). Notons que si $\mathbf{I}$ est en retard sur $\mathbf{E}$ (amplitude de $\left.\mathbf{E}: E=\phi_{f} \cdot \omega\right), \psi$ est positif ( $I_{\mathrm{d}}>0$, magnétisant), sinon il est négatif $\left(I_{\mathrm{d}}<0\right.$, démagnétisant). Toutes les grandeurs $I, V$ et $E$ considérées sont des valeurs efficaces.

L'équation d'alimentation est :

$$
V^{2}=\left(E+L \cdot \omega \cdot I_{\mathrm{d}}\right)^{2}+\left(L \cdot \omega \cdot I_{\mathrm{q}}\right)^{2} \text { avec } E=\phi_{\mathrm{f}} \cdot \omega
$$

$V$ est la tension fondamentale simple, $L$ est l'inductance cyclique. On constate qu'à angle $\psi$ et amplitude du courant fixés, la tension d'alimentation est proportionnelle à la vitesse. Aussi, dès que la tension maximale disponible est atteinte, si la vitesse croît, l'amplitude du courant qu'il est possible de réguler diminue et le couple diminue très rapidement. Autrement dit, le moteur est capable de délivrer son couple maximal (courant maximal $I_{\text {Max }}=I$ ) jusqu'à une vitesse de base $\Omega_{\mathrm{b}}=\frac{\omega_{\mathrm{b}}}{p}$ ( $p$ est le nombre de paires de pôles) telle que :

$$
\Omega_{\mathrm{b}}=\frac{V}{p \cdot \sqrt{\left(\phi_{\mathrm{f}}+L \cdot I_{\mathrm{d}}\right)^{2}+\left(L \cdot I_{\mathrm{q}}\right)^{2}}}
$$

Pour une machine à pôles lisses, les pertes Joule sont minimales si $\psi=0$; si cette stratégie est adoptée la composante directe du courant est nulle : 
Si $\psi=0: I_{\mathrm{d}}=0$ et $I_{\mathbf{q}}=I$, alors :

$$
p \cdot \Omega_{\mathrm{b}}=\frac{V}{\sqrt{\left(\phi_{\mathrm{f}}\right)^{2}+(L \cdot I)^{2}}}
$$

À la vitesse de base et au couple "nominal", le facteur de puissance $\cos \varphi_{\mathrm{b}}$ vaut alors :

$$
\cos \varphi_{\mathrm{b}}=\cos \left[\operatorname{Arctg} \frac{L \cdot I}{\phi_{\mathrm{f}}}\right]
$$

On remarque que le facteur de puissance est d'autant plus proche de 1 que l'inductance de la machine est faible (aimants montés en surface), en fait, il faut que le flux de réaction d'induit soit faible devant le flux inducteur.
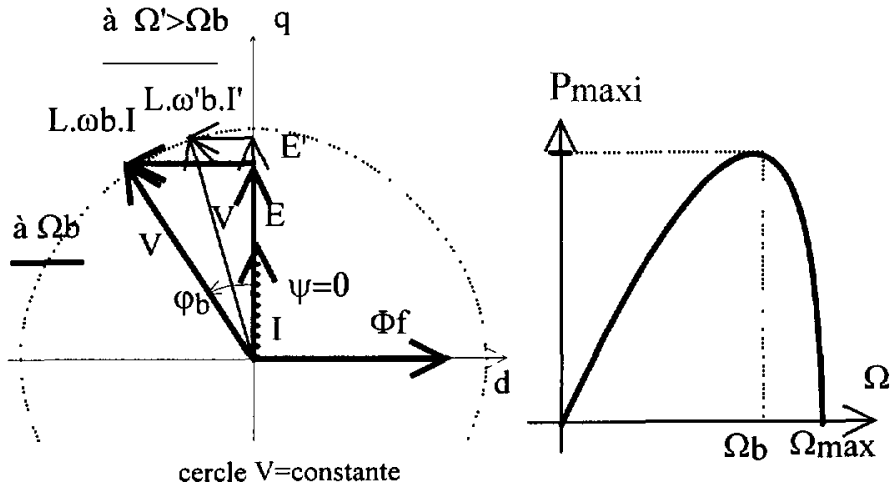

Fig. 2. - Evolution du diagramme vectoriel à une vitesse supérieure à la vitesse de base, à $\psi=0$. La puissance maximale décroît rapidement.

[Evolution of the vector diagram at speed higher base speed with $\psi=0$. The maximum power drastically decreases.]

Comme le montre le diagramme vectoriel de la figure 2, si la vitesse dépasse la vitesse de base, le courant et, donc, le couple décroissent. En particulier, lorsque $E=V$, le courant devient nul, pour une vitesse maximale égale à :

$$
\Omega_{\mathrm{Max}}=\frac{V}{E_{\mathrm{b}}} \cdot \Omega_{\mathrm{b}}=\frac{1}{\cos \varphi_{\mathrm{b}}} \cdot \Omega_{\mathrm{b}}
$$

Au delà de la vitesse de base, le couple décroît très vite et il en est de même de la puissance. Aussi, si l'on souhaite augmenter la puissance convertible pour des vitesses supérieures à la vitesse de base, il faut accepter de faire évoluer l'angle d'autopilotage $\psi$.

On peut remarquer que si $\psi$ est négatif ( $I$ en avance sur $E$ ), on réduit le flux résultant ainsi que la tension induite totale, égale à $V$ lorsque l'on néglige la chute ohmique. 
Lorsque le moteur est alimenté sous tension constante (la tension maximale disponible), la puissance peut s'exprimer par :

$$
P=\frac{3}{L \cdot \omega} \sqrt{4 \cdot(V \cdot E)^{2}-\left(V^{2}+E^{2}-(L \cdot \omega \cdot I)^{2}\right)^{2}}
$$

En posant :

$$
\begin{gathered}
r=\frac{L \cdot I}{\phi_{\mathrm{f}}} \text { la réaction d'induit relative, } \\
x=\frac{\omega}{\omega_{\mathrm{b}}}=\frac{N}{N_{\mathrm{b}}} \text { la vitesse réduite } \\
e=\frac{E_{\mathrm{b}}}{V}=\sqrt{\frac{1}{1+r^{2}}} \text { la f.e.m. à la vitesse de base réduite }
\end{gathered}
$$

on obtient :

$$
\frac{P}{3 \cdot V \cdot I}=\frac{1}{2 \cdot e \cdot r \cdot x} \sqrt{4 \cdot(e \cdot x)^{2}-\left(1+(e \cdot x)^{2}-(r \cdot e \cdot x)^{2}\right)^{2}}
$$

Cette expression représente la puissance maximale convertible, en fonction de la vitesse à contraintes d'alimentation données ; c'est aussi le facteur de puissance. Notons que, lorsque $r<1$, la puissance maximale, au delà de la vitesse de base $(x>1)$, est limitée par le courant maximal (voir paragraphe 3.2, Fig. 8) et que le calcul est donc effectué à $I=\mathrm{Cte}$. On peut en effet démontrer qu'il est possible d'atteindre $I$ sous la tension $V$ quelle que soit la vitesse. Théoriquement, à tension maximale donnée, la puissance est maximale lorsque l'angle de charge $\delta$ (entre $V$ et $E$ ) est égal à $\pi / 2$, mais alors le courant est toujours supérieur à $I$, valeur "de base" ; ce régime est donc inacceptable.

La figure 3a montre l'importance de $r$ pour obtenir une large plage de vitesse à puissance élevée. On n'a pas représenté ce qui se passe si $r>1$, la puissance est alors toujours plus faible et ce cas ne présente pas d'intérêt.

La figure $3 \mathrm{~b}$ montre le réseau, paramétré en $r$, du couple normalisé disponible, en fonction de la vitesse réduite $x$.

Le cas $r=1$, permet d'avoir une vitesse maximale "illimitée" et correspond à un cas idéal de fonctionnement à puissance constante. On peut remarquer que le facteur de puissance se rapproche de 1 au delà de 3 fois la vitesse de base.

Le facteur de puissance à la vitesse de base (où $\psi=0$ ) vaut :

$$
\cos \varphi_{\mathrm{b}}=\frac{1}{\sqrt{1+r^{2}}}
$$

Donc pour $r=1, \cos \varphi_{\mathrm{b}}=0,707$.

La figure $4 \mathrm{a}$ montre comment évolue le diagramme vectoriel (à la vitesse de base : cas identique à celui de la Fig. 2). Le point de fonctionnement représenté au delà de la vitesse de base correspond à la puissance maximale (facteur de puissance unitaire). De la vitesse de base jusqu'à la vitesse (en valeur réduite : $x_{P_{\max }}$ ) correspondant à la puissance maximale, le courant est, dans ce cas, en retard sur la tension, au delà il est en avance. La figure 4b met en évidence l'évolution de l'angle d'avance $(\psi<0)$ du courant par rapport à la f.e.m. Celui-ci part de 0 , pour les vitesses inférieures à la vitesse de base, pour aller vers $\pi / 2$ à la vitesse maximale possible en régime de "défluxage" $x_{\mathrm{Mfv}}$. 


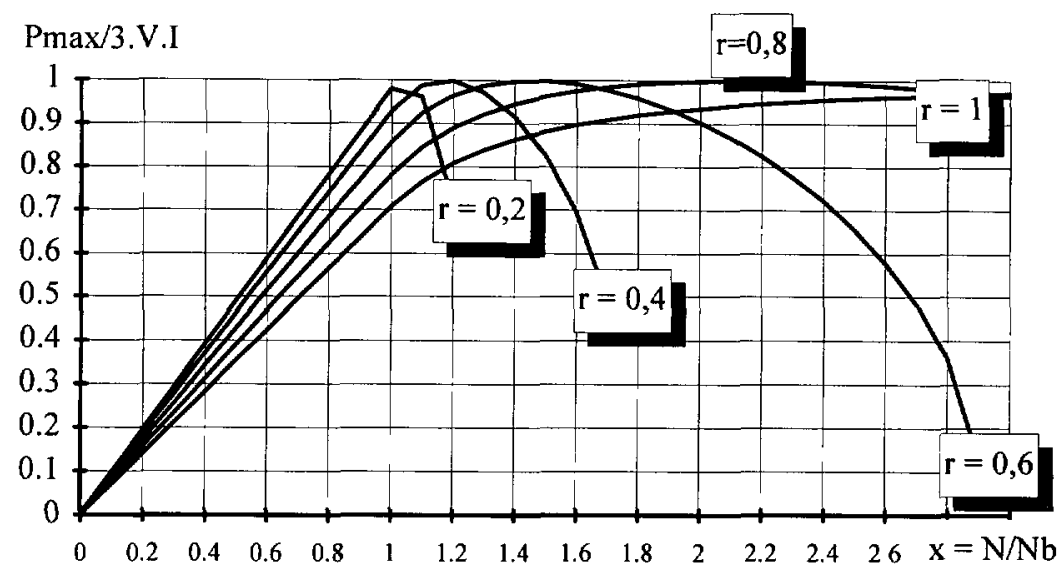

Cmax

a)

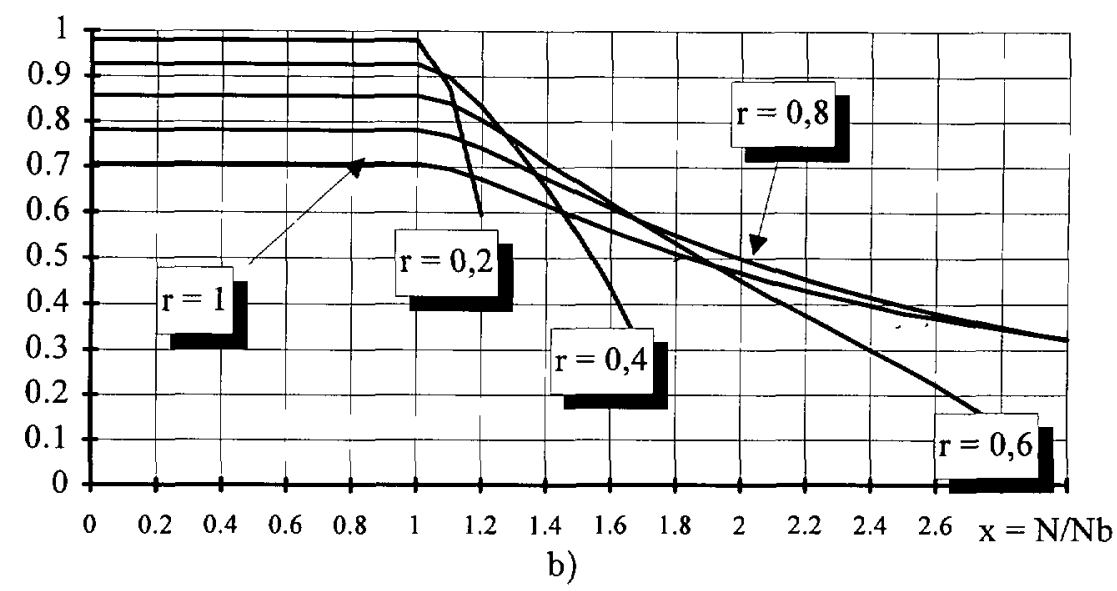

Fig. 3. - a) Puissance maximale disponible en fonction de la vitesse normalisée $x$. Le paramètre $r$ est la réaction d'induit réduite (Eq. 3.8). b) Couple maximal en fonction de la vitesse normalisée.

(a) Maximum power versus normalised speed. Parameter $r$ is the normalised armature reaction (Eq. 3.8). b) Maximum torque versus normalised speed.]

Notons que cette vitesse maximale (à $P=0$ ) en régime de "désexcitation" est maintenant définie par $\psi=\pi / 2$, on obtient alors en valeurs réduites :

$$
x_{\mathrm{Mfv}}=\frac{\sqrt{1+r^{2}}}{1-r}
$$

Rappelons que, en gardant $\psi=0$ (pas de désexcitation), d'après (3.6), on avait :

$$
x_{\mathrm{M} \psi=0}=\sqrt{1+r^{2}}
$$

La vitesse maximale est donc accrue dans le rapport $\frac{1}{1-r}$, rapport d'autant plus grand que la réaction d'induit relative $r$ est élevée. 


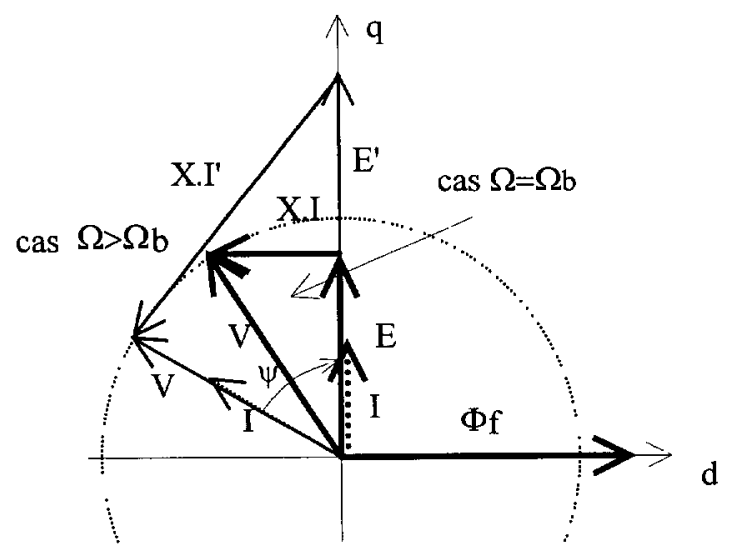

cercle $\mathrm{V}=$ constante

a)

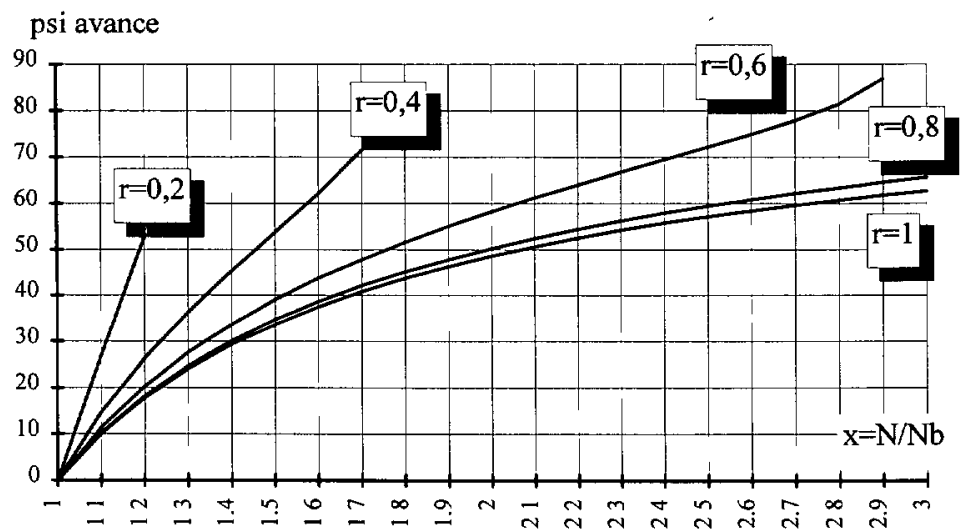

b)

Fig. 4. - a) Diagramme de Fresnel en régime de désexcitation $(\psi<0)$. b) Angle d'avance $(-\psi)$ en fonction de la vitesse normalisée en régime de désexcitation.

[a) Vector diagram in flux-weakening mode $(\psi<0)$. b) Advance angle $(-\psi)$ versus normalised speed in flux-weakening mode.]

La puissance maximale, quant à elle, est atteinte à une vitesse telle que le déphasage courant tension est nul, on obtient alors :

$$
x_{\mathbf{P}_{\max }}=\sqrt{\frac{1+r^{2}}{1-r^{2}}}
$$

Enfin, on peut rechercher dans quelle plage de vitesse, il est possible de maintenir une puissance supérieure ou égale à celle atteinte à la vitesse de base. La vitesse réduite correspondant à cette plage de fonctionnement à "puissance sensiblement zonstante" est notée $x_{\text {PMC }}$. Elle peut être déterminée par le point de fonctionnement tel que $\cos \varphi=\cos \varphi_{\mathrm{b}}$ avec le courant en avance sur la tension. Alors on obtient : 


$$
x_{\mathrm{PMC}}=\frac{1+r^{2}}{1-r^{2}}
$$

La figure 5 montre les courbes d'évolution, en fonction de la réaction d'induit nominale relative $r$, des vitesses réduites $x_{\mathrm{M} \psi=0}$ (courbe 1, vitesse maxi possible sans "désexcitation"), $x_{\text {Mfv }}$ (courbe 4 , vitesse maximale possible avec "désexcitation"), $x_{P_{\max }}$ (courbe 2, vitesse correspondant à la puissance maximale) et $x_{\mathrm{PMC}}$ (courbe 3 , vitesse maximale définissant la plage à "puissance constante"). Par exemple, si l'on souhaite une plage à puissance constante dans un rapport 1 à 5 , il est nécessaire que $r>0,82$.

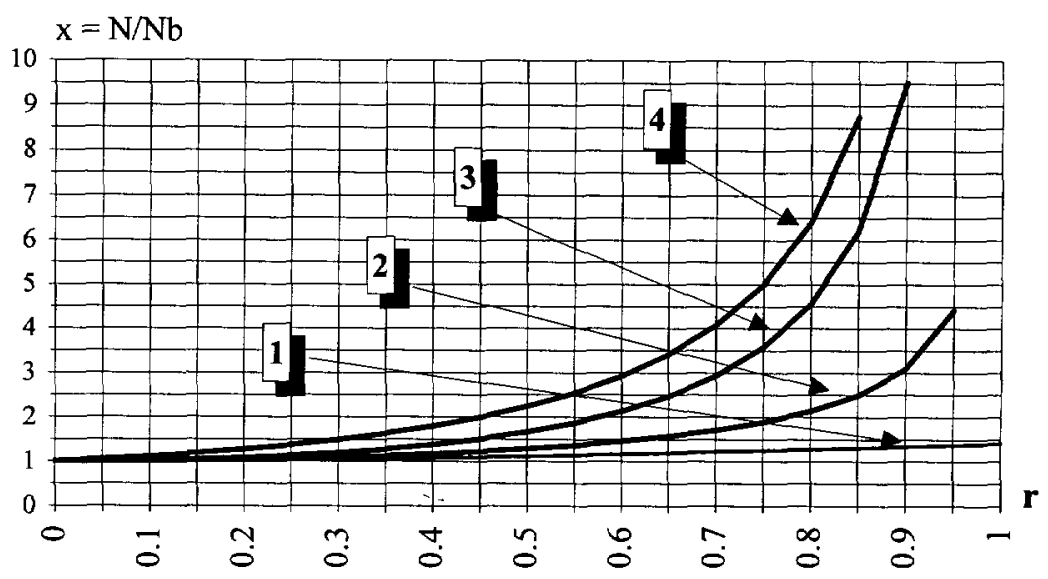

Fig. 5. - Vitesses maximales possibles sans désexcitation (1), avec désexcitation (4), à puissance maximale $(\cos \varphi=1)(2)$ et plage de vitesses à puissance constante $(3)$.

[Maximum speeds with (4) and without field-weakening (1), speed at maximum power $(\cos \varphi=1)(2)$ and constant power speed range (3).]

Ainsi, pour obtenir une possibilité de fonctionnement à puissance élevée (voire constante) audelà de la vitesse de base, il est nécessaire d'optimiser la valeur de l'inductance par rapport au flux inducteur. La condition " $r$ voisin de 1 " est difficile, voire impossible, à obtenir avec un rotor à aimants montés en surface ; il faut donc des structures à pièces polaires, généralement à aimants enterrés. La contrepartie de cette réaction d'induit élevée est un plus faible facteur de puissance à la vitesse de base.

En outre, la réaction d'induit démagnétisante ne doit pas désaimanter de manière irréversible les aimants. Les pièces polaires peuvent ainsi permettre au flux de réaction d'induit de ne pas "traverser" les aimants.

Si des structures à aimants enterrés sont préférables, on ne peut plus faire l'hypothèse de pôles lisses. Il faut donc faire une étude avec $L_{\mathrm{d}}$ et $L_{\mathrm{q}}$ différentes.

3.2. Machines aVEC Saillance. - Le couple moyen s'exprime alors par :

$$
T=3 \cdot\left[\phi_{\mathrm{f}} \cdot I_{\mathrm{q}}+\left(L_{\mathrm{d}}-L_{\mathrm{q}}\right) \cdot I_{\mathrm{d}} \cdot I_{\mathrm{q}}\right]
$$


Pour minimiser les pertes Joule, l'angle d'autopilotage optimal vaut :

$$
\begin{gathered}
\text { si } L_{\mathrm{d}}>L_{\mathrm{q}} \quad \psi_{\mathrm{opt}}=\operatorname{Arcsin}\left[\frac{-k+\sqrt{k^{2}+8}}{4}\right]>0 \text { avec } k=\frac{\phi_{\mathrm{f}}}{\left(L_{\mathrm{d}}-L_{\mathrm{q}}\right) \cdot I} \\
\text { si } L_{\mathrm{d}}<L_{\mathrm{q}} \psi_{\mathrm{opt}}=\operatorname{Arcsin}\left[\frac{-k-\sqrt{k^{2}+8}}{4}\right]<0
\end{gathered}
$$

La réaction d'induit normalisée est maintenant définie par :

$$
r=\frac{L_{\mathrm{d}} \cdot I}{\phi_{\mathrm{f}}}
$$

On définit le rapport de saillance :

$$
s=\frac{L_{\mathrm{q}}}{L_{\mathrm{d}}}
$$

Dans le cas particulier où $r=1$ (cas idéal de la désexcitation), la valeur de $\psi_{\text {opt }}$ est donnée sur la figure 6 .

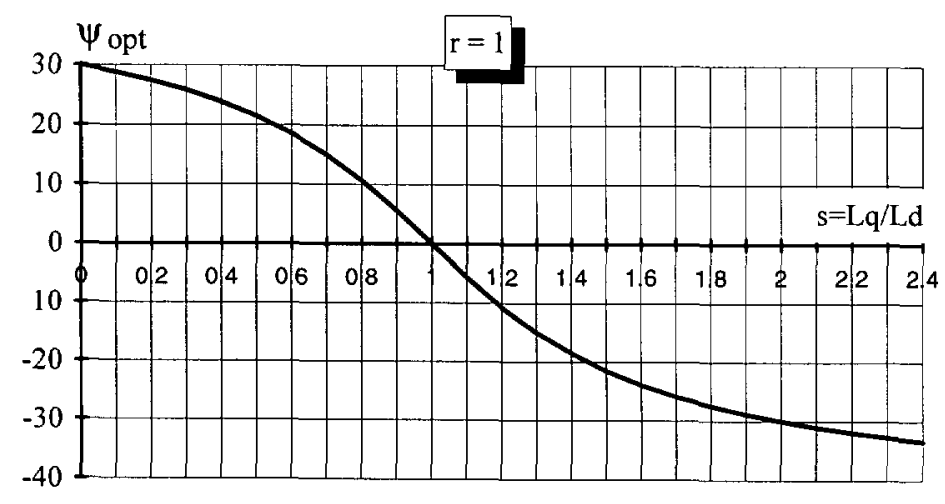

Fig. 6. - Angle d'autopilotage optimal $\left(\psi_{\mathrm{opt}}\right)$ à la vitesse de base en fonction du rapport de saillance $s$ et pour $r=1$.

[Optimum $\left(\psi_{\text {opt }}\right)$ angle at base speed versus saliency ratio $s$ and for $r=1$.]

Ainsi pour une machine dans laquelle $L_{\mathrm{d}}>L_{\mathrm{q}}(s<1)$, l'angle $\psi$ d'autopilotage optimal (pour $r=1$ ) est positif et tend vers $30^{\circ}$ lorsque $s$ tend vers 0 . La réaction d'induit est alors magnétisante. Dans le cas contraire (le plus fréquent dans les machines à aimants enterrés), la réaction d'induit est démagnétisante et l'angle optimal tend vers $45^{\circ}$ lorsque $s$ tend vers l'infini.

En outre, le facteur de puissance $\cos \varphi$ à la vitesse de base dépend de $s$ et peut être amélioré par rapport au cas sans saillance $(s=1)$ où il valait 0,707 lorsque $r=1$. Si $s$ tend vers $0, \cos \varphi$ tend vers 0,866 et lorsque $s$ tend vers l'infini, il tend vers 0,707 ; pour $s>1$, il passe par un maximum $(0,72)$ au voisinage de $s=2$. Il semble ainsi plus intéressant d'avoir $s<1$.

La figure 7 montre l'évolution du facteur de puissance (3.19) et du gain sur le couple $G_{c}$ (3.20) par rapport à un fonctionnement à $I_{\mathrm{d}}=0(\psi=0)$ en fonction du rapport de saillance $s$ : 


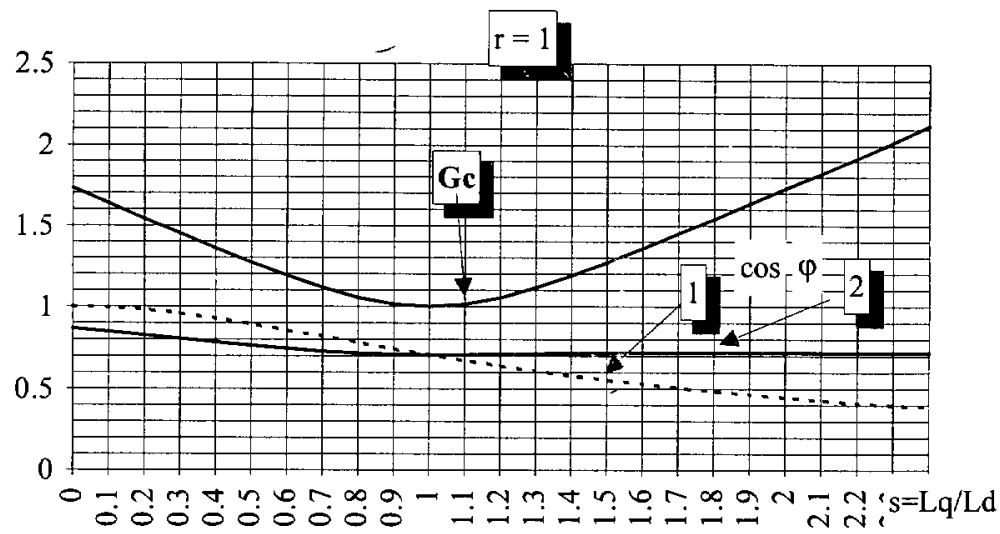

Fig. 7. $-\psi=\psi_{\mathrm{opt}}$ : Gc gain sur le couple par rapport à une commande à $\psi=0$. Facteur de puissance à la vitesse de base (courbe 2) $(\psi=0$ : courbe 1$)$.

$\left[\psi=\psi_{\mathrm{opt}}\right.$. increasing of torque in regard to the case $\psi=0$. Power factor at base speed (curve 2), $(\psi=0$ : curve 1$)$.]

$$
\begin{gathered}
\varphi=\operatorname{Arctg}\left[\frac{L_{\mathrm{q}} \cdot I \cdot \cos \psi_{\mathrm{opt}}}{\phi_{\mathrm{f}}+L_{\mathrm{d}} \cdot I \cdot \sin \psi_{\mathrm{opt}}}\right]+\psi_{\mathrm{opt}}=\operatorname{Arctg}\left[\frac{r \cdot s \cdot \cos \psi_{\mathrm{opt}}}{1+r \cdot \sin \psi_{\mathrm{opt}}}\right]+\psi_{\mathrm{opt}} \\
G_{\mathrm{c}}=\cos \psi_{\mathrm{opt}}+\frac{r}{2} \cdot(1-s) \cdot \sin \left(2 \cdot \psi_{\mathrm{opt}}\right)
\end{gathered}
$$

La figure 8 représente les différentes limites du trajet de l'extrémité du vecteur I dans le plan $\left(I_{\mathrm{d}}, I_{\mathrm{q}}\right)$. Le courant est limité en amplitude, d'une part, par la valeur maximale (pertes Joule, courant commutable par l'onduleur) qui donne un cercle limite, d'autre part, par la tension maximale disponible et la vitesse qui conduisent à des ellipses dont la plus grande correspond à la vitesse de base.

Sur cette figure, on a représenté le cas (peu fréquent) où $r>1$ (le centre des ellipses, point Ov, se trouve à l'intérieur du cercle de courant maxi) et où $s>1$ ( $\psi<0$ en dessous de la vitesse de base).

L'équation suivante décrit ces ellipses :

$$
V=\omega \cdot \sqrt{\left(\phi_{\mathrm{f}}+L_{\mathrm{d}} \cdot I_{\mathrm{d}}\right)^{2}+\left(L_{\mathrm{q}} \cdot I_{\mathrm{q}}\right)^{2}}
$$

Les points A, B, C, D et Ov (Fig.8) ont respectivement pour coordonnées dans le plan $\left(I_{\mathrm{d}}, I_{\mathrm{q}}\right)$ :

$$
\begin{array}{ll}
\mathrm{A}:\left(-\frac{V}{L_{\mathrm{d}} \cdot \omega_{\mathrm{b}}}-\frac{\phi_{\mathrm{f}}}{L_{\mathrm{d}}}, 0\right) & \mathrm{B}:\left(+\frac{V}{L_{\mathrm{d}} \cdot \omega_{\mathrm{b}}}-\frac{\phi_{\mathrm{f}}}{L_{\mathrm{d}}}, 0\right) \\
\mathrm{C}:\left(-\frac{\phi_{\mathrm{f}}}{L_{\mathrm{d}}}, \frac{V}{L_{\mathrm{q}} \omega_{\mathrm{b}}}\right) & \mathrm{D}:\left(0, \sqrt{\left(\frac{V}{L_{\mathrm{q}} \cdot \omega_{\mathrm{b}}}\right)^{2}-\left(\frac{\phi_{\mathrm{f}}}{L_{\mathrm{q}}}\right)^{2}}\right) \\
\mathrm{Ov}:\left(-\frac{\phi_{\mathrm{f}}}{L_{\mathrm{d}}}, 0\right) &
\end{array}
$$

Pour tirer le meilleur parti des caractéristiques de l'ensemble convertisseur-moteur, on peut adopter la stratégie suivante (Fig. 8, 9) [10] : 


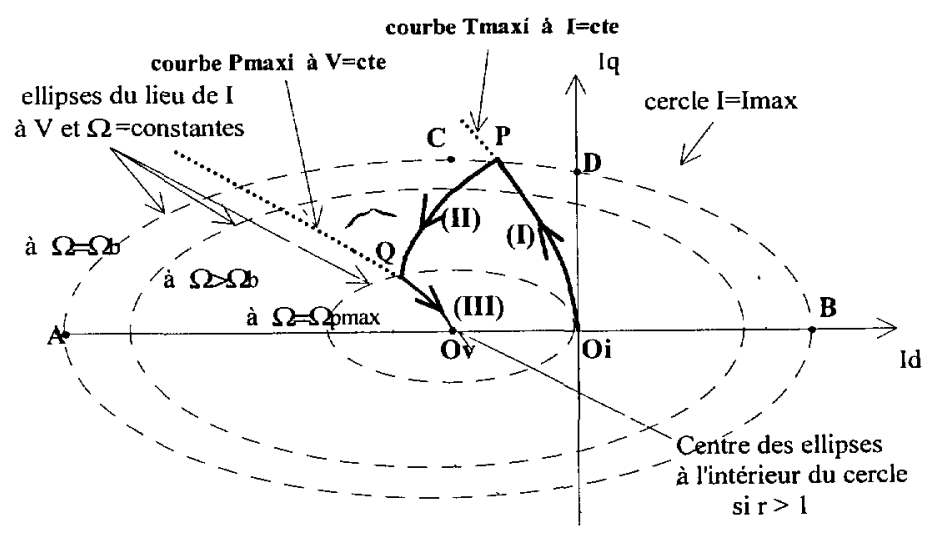

Fig. 8. - Limites du trajet de l'extrémité du vecteur $\mathbf{I}$ dans le plan $\left(I_{\mathrm{d}}, I_{\mathrm{q}}\right)$.

[Limits of the I vector locus due to maximum values of $I$ (circle) and $V$ (ellipses) $\left(\left(I_{\mathrm{d}}, I_{\mathrm{q}}\right)\right.$ plane.]

- lorsque $\Omega \leq \Omega_{\mathrm{b}}$, (zone (I) de Oi à $\mathrm{P}$, figure 8) on maximise le couple ( $\psi=\psi_{\text {opt }}$ ) ;

- lorsque $\Omega>\Omega_{\mathrm{b}}$, on déphase le courant pour travailler à courant maximal (cercle $I_{\max }$ ) tant que la tension maximale le permet (zone (II)) pour obtenir la puissance la plus élevée possible. Si $r>1$ (point $\mathrm{Ov}$ à l'intérieur du cercle de courant maxi), une troisième zone apparait : à partir d'une vitesse $\Omega_{p_{\max }}$ (point $Q$, figure 8 ) on ne peut plus réguler le courant à sa valeur maximale et l'on cherche seulement à maximiser la puissance à tension maximale, le trajet de l'extrémité du vecteur I suit alors le lieu de puissance maximale à $V=$ Cste (zone (III)). Les équations de $\left(I_{\mathrm{d}}, I_{\mathrm{q}}\right)$ en fonction de $\omega$ à puissance maximisée et à $V=$ Cste sont, d'après [10] :

$$
\left(-\frac{\phi_{\mathrm{f}}}{L_{\mathrm{d}}}-\Delta i_{\mathrm{d}}, \sqrt{\left(\frac{V}{L_{\mathrm{q}} \cdot \omega}\right)^{2}-\left(\frac{\Delta i_{\mathrm{d}}}{s}\right)^{2}}\right)
$$

avec $\Delta i_{\mathrm{d}}=0$ si $s=1$ et

$$
\Delta i_{\mathrm{d}}=\frac{-s \cdot \phi_{\mathrm{f}}+\sqrt{\left(s \cdot \phi_{\mathrm{f}}\right)^{2}+8 \cdot(s-1)^{2} \cdot\left(\frac{V}{\omega}\right)^{2}}}{4 \cdot(s-1) \cdot L_{\mathrm{d}}} \text { si } s \neq 1
$$

La figure 10 montre l'influence du rapport de saillance $s$ sur le facteur de puissance en régime de désexcitation lorsque l'on respecte la condition de désexcitation idéale $(r=1)$. On constate que le cas $s>1$ donne une légère amélioration du facteur de puissance par rapport au cas sans saillance et que le cas $s<1$ permet un gain tout à fait intéressant, d'environ $20 \%$ lorsque $L_{\mathrm{d}}=4 L_{\mathrm{q}}$. Lorsque $s<0,5$, le facteur de puissance passe par un maximum ( $\cos \varphi \cong 1$ pour $s=0,25$ à $x \cong 2,1)$ puis décroît vers un minimum $\operatorname{local}(\cos \varphi \cong 0,956$ pour $s=0,25$ à $x \cong 8,5$ ) pour tendre à nouveau vers 1 aux vitesses très supérieures. Plus $s$ est faible plus le facteur de puissance passe par un minimum local faible. Il faut donc éviter les très faibles valeurs de $s(<0,2)$.

Le couple de paramètres $r=1$ et $s=0,25$ permet d'avoir un bon facteur de puissance (supérieur à 0,82 ) sur une plage de vitesse théoriquement infinie et nous semble offrir les meilleures conditions de "fonctionnement à puissance constante". Nous verrons au dernier paragraphe que la plupart des structures de rotor permettant d'atteindre la condition $r=1$ conduisent malheureusement à un rapport $s$ supérieur à 1 . 


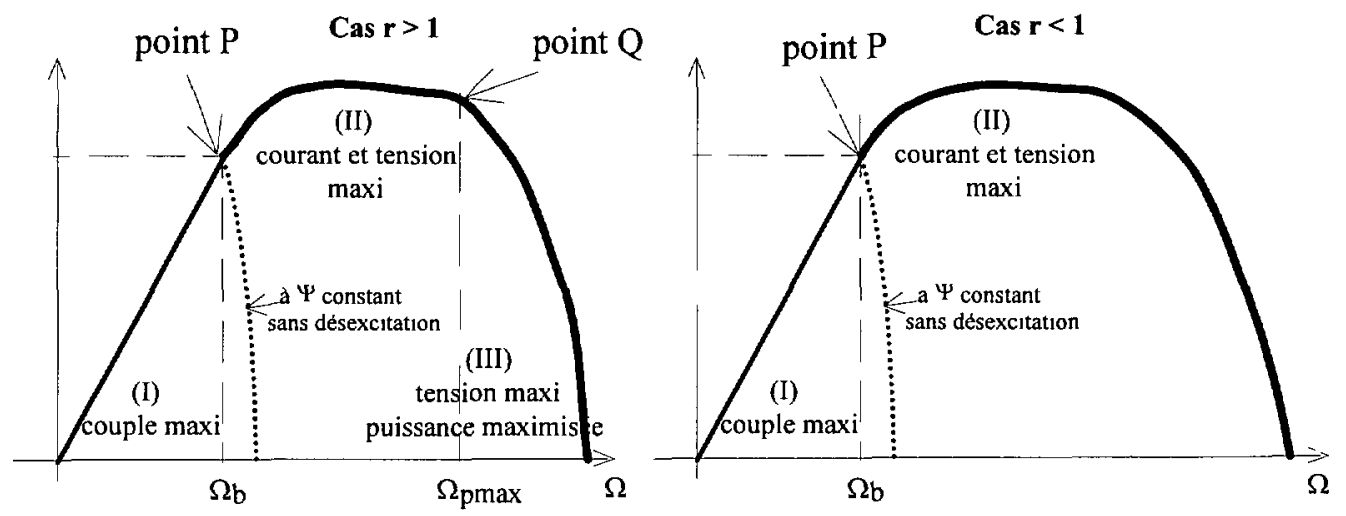

Fig. 9. - Allures (dans les cas $r>1$ et $r<1$ ) de la courbe de puissance maximale en fonction de la vitesse correspondant à la figure 8 .

[Forms (in the cases $r>1$ et $r<1$ ) of the maximum power curve versus speed corresponding to Figure 8.]

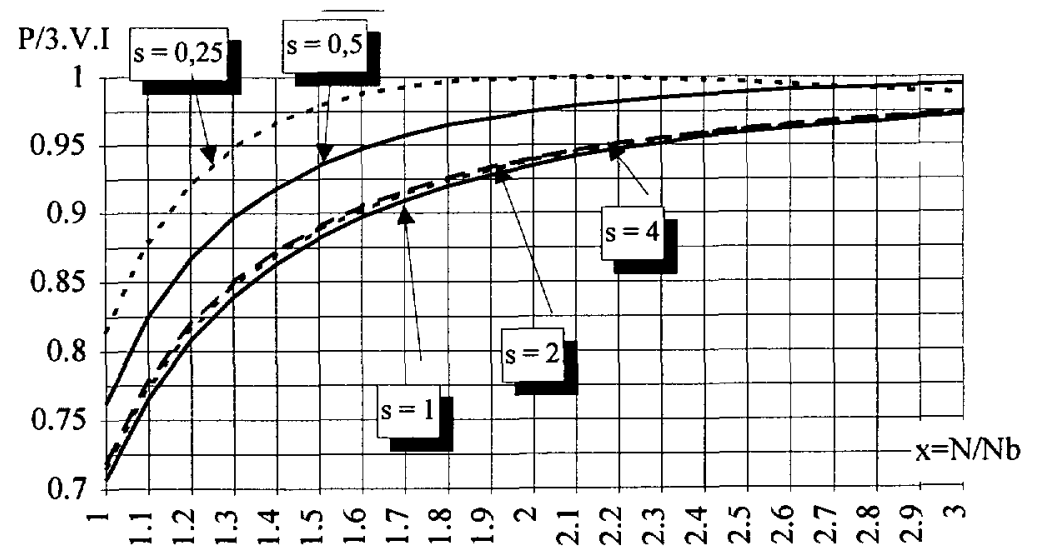

Fig. 10. - Facteur de puissance (ou puissance maximale normalisée) en fonction de la vitesse lorsque $r=1$, le paramètre $s$ est le rapport de saillance.

[Power factor versus speed when $r=1, s$ parameter is the saliency ratio.]

Les courbes de la figure 10 ont été obtenues à partir de l'expression (3.24) dans laquelle $\psi_{0}$ est solution de l'équation (3.25) correspondant à un fonctionnement à $I$ et $V$ constants et $\psi_{\text {opt }}$ est la valeur optimale choisie pour minimiser les pertes Joule à la vitesse de base (expression $(3.16))$.

$$
\begin{gathered}
\frac{P}{3 \cdot V \cdot I} \cdot \cos \varphi=e \cdot x \cdot \cos \psi_{0} \cdot\left[1+r \cdot(1-s) \cdot \sin \psi_{0}\right] \\
\text { avec : } e=\left[\left(1+r \cdot \sin \psi_{\mathrm{opt}}\right)^{2}+\left(s \cdot r \cdot \cos \psi_{\mathrm{opt}}\right)^{2}\right]^{-0.5} \\
r^{2} \quad\left(1-s^{2}\right) \cdot \sin ^{2} \psi+2 \cdot r \cdot \sin \psi+1+(s \cdot r)^{2}-(e \cdot x)^{-2}=0
\end{gathered}
$$


Globalement, la présence d'une saillance $(s \neq 1)$ permet de trouver un meilleur compromis entre le facteur de puissance à la vitesse de base et la plage de vitesse à puissance maximale élevée voire constante.

\section{Structures de rotors aptes à la désexcitation}

Il apparaît de façon évidente que la conception du rotor à aimants permanents est de première importance quant à l'obtention d'une machine à commutation électronique à bon facteur de puissance et à large plage à puissance maximale constante.

La figure 11 récapitule quelques unes des principales structures d'inducteurs réalisées et/ou proposées. Le rotor à aimants permanents en surface, qui conduit à une inductance sensiblement constante $(s=1)$ et à une faible réaction d'induit ( $r$ faible), n'a pas été représenté et ne permet pas de satisfaire les conditions de désexcitation.

La figure 11a montre un rotor à aimants insérés [4,19,22] (les aimants sont aimantés radialement et sont insérés dans des tôles empilées ou dans un rotor massif) : $s>1$. Cet agencement permet une désexcitation de qualité moyenne, dans la référence [4], une plage de vitesse en régime "Flux Weakening" de 1 à 4 a été atteinte.

La figure 11b montre une nouvelle disposition [22] permettant d'avoir $s<1\left(L_{\mathrm{q}}<L_{\mathrm{d}}\right)$, donc un bon facteur de puissance, une réaction d'induit relative $r$ suffisante et enfin de protéger les aimants contre la désaimantation. Cette solution semble l'une des meilleures mais elle n'est pas simple à réaliser.

La figure 11c montre une structure à aimants enterrés et à "concentration de flux" $[1,5,20]$, elle permet d'avoir un flux par pôle élevé. Mais l'obtention de bonnes caractéristiques pour le fonctionnement en régime de désexcitation dans une large plage est difficile. Dans la référence [20], une analyse des "longueurs" d'entrefer et d'aimants est effectuée et met en évidence l'intérêt d'un faible entrefer qui permet d'accroître plus vite la réaction d'induit que le flux, donc d'augmenter le rapport $r$. L'épaisseur des aimants ne doit pas être trop grande pour trouver un juste compromis entre le flux et le rapport $r$. Dans la reférence [20], une plage de vitesse à puissance élevée d'un rapport 1:3,5 est obtenue.

La figure $11 \mathrm{~d}$ montre une structure proposée pour un moteur à très grande plage à puissance maximale constante (1:10) [24]. Ce type de rotor permet d'obtenir, semble-t-il, à la fois un flux élevé par pôle et une réaction d'induit suffisante.

La figure 11e décrit la structure appelée "I.P.M." ("Interior Permanent Magnet" dans la littérature) $[2-4,7,10,17]$ et abondamment étudiée pour l'obtention d'un fonctionnement à puissance constante. Le rapport de saillance $s$ est supérieur à 1 . De grandes plages de vitesse en régime de désexcitation ont été obtenues $[3,7,10]$ dans des rapports de 1 à 4 environ.

Le rotor de la figure $11 \mathrm{f}$ est un rotor de moteur synchrone à réluctance variable dans lequel on a rajouté des aimants $[12,14,16]$, les auteurs qualifient cette structure de "Permanent Magnet Assisted Synchronous Reluctance" (P.M.A.S.R.). Dans la référence [16] on obtient : $s \approx 10$ (Attention, dans les articles cités, l'axe $q$ correspond à celui d'inductance mini et ici, à celui du flux inducteur !).

Dans la référence [28], c'est une structure de type synchrone à réluctance à rotor "axialement laminé" qui est assistée par des aimants ferrites fins à liant plastique (souples, $B_{\mathrm{r}}=0,165 \mathrm{~T}$ ) logés entre les tôles empilées axialement. Une plage à puissance constante dans un rapport supérieur à 7,5 est obtenue avec un facteur de puissance de 0,8 à la vitesse de base. Ces dernières machines sont alors plutôt considérées comme des moteurs synchrones à réluctance variable dans lesquels il est possible d'obtenir une plage à puissance maximale constante "infinie" en compensant le flux dans l'axe $q$ grâce à des aimants $\left(\left|L_{\mathbf{q}} \quad I\right|=\phi_{\mathrm{f}}\right)$. 


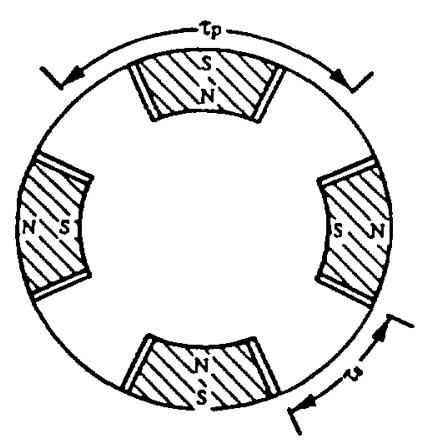

a

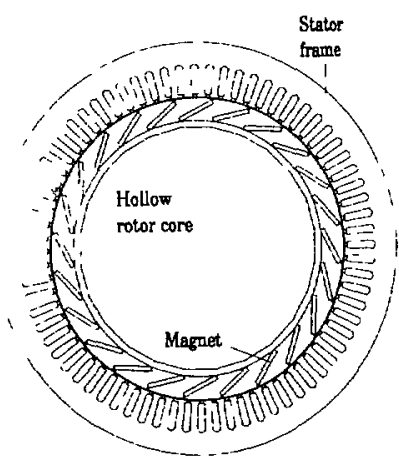

d

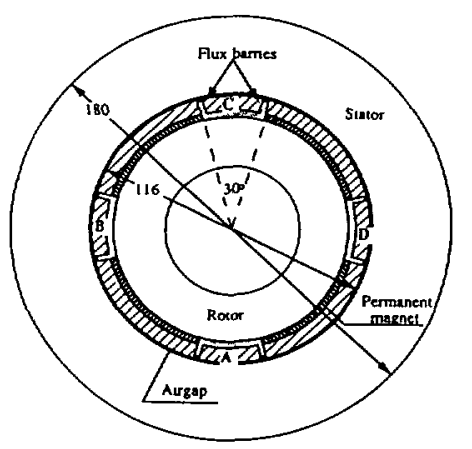

b

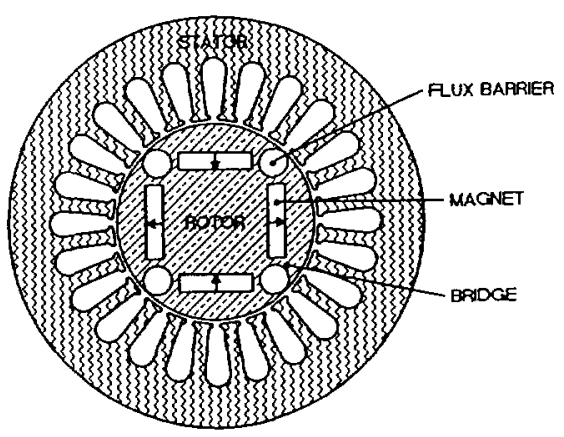

$\mathrm{e}$

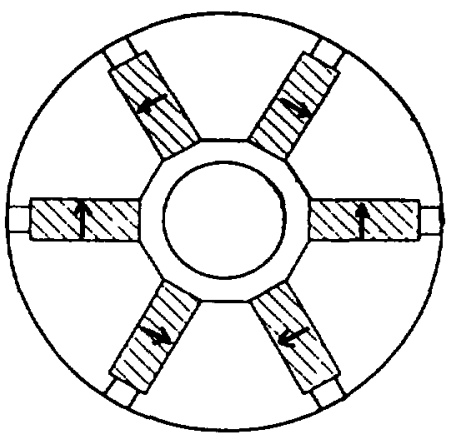

c

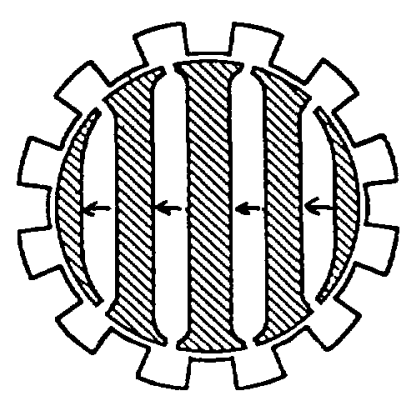

f

Fig. 11. - Structures de rotor permettant le fonctionnement en régime de désexcitation. [Permanent magnet rotor structures permitting flux-weakening operation.]

Notons qu'il existe d'autres types de moteurs spéciaux à aimants, par exemple dans les références $[11,26,27,29]$, qui permettent plus ou moins bien le fonctionnement en régime de désexcitation. Mêmes s'ils ne sont pas conventionnels (pas de champ tournant), les règles de désexcitation sont les mêmes que celles des moteurs à aimants plus classiques.

\section{Conclusion}

Dans cet article, nous avons mis en évidence les limites d'alimentation des machines à aimants permanents alimentées par onduleur, plus particulièrement en courants sinusoïdaux régulés par modulation de largeur d'impulsion. Les hypothèses de calcul concernant la machine sont très simplistes (f.e.m. sinusoïdales, induction sinusoïdale dans l'entrefer), elles ont permis une étude paramétrique assez simple, générale et toutefois convenable. Mais il faudrait, dans des cas particuliers, déterminer précisément les caractéristiques électromagnétiques réelles et effectuer des simulations numériques d'alimentation. Nous avons ensuite analysé l'influence des paramètres de la machine (flux inducteur et inductance) dans le cas particulier d'une inductance constante ("pôles lisses"). Nous avons montré que plus le flux de réaction d'induit nominal $(L \cdot I)$ était élevé par rapport au flux inducteur, plus grande était la plage de fonctionnement à puissance constante, toutefois, il ne faut pas que l'amplitude de ce flux dépasse celle du flux 
inducteur. S'il y a égalité entre ces deux flux, on obtient une plage de "désexcitation" infinie. La "désexcitation" est obtenue grâce au déphasage en avance du courant d'induit par rapport à la force électromotrice qui permet la création d'une composante de force magnétomotrice démagnétisante. Dans le cas "idéal" de la plage de fonctionnement à puissance constante infinie, le facteur de puissance à la vitesse de base est malheureusement faible $(0,707)$ aussi il est nécessaire de rechercher un compromis entre un bon facteur de puissance à la vitesse de base et la plage de fonctionnement à puissance maximale nécessaire pour l'application envisagée. Ensuite, nous avons étudié le cas des rotors magnétiquement anisotropiques ("pôles saillants") qui permettent aussi le "défluxage" tout en améliorant le facteur de puissance, et bien sûr le couple moyen, par rapport aux rotors "lisses". Nous avons montré qu'il était particulièrement intéressant d'obtenir $L_{\mathbf{d}}>L_{\mathbf{q}}(s<1)$ pour avoir un gain significatif du facteur de puissance.

Enfin, nous avons passé en revue quelques structures de rotors à aimants dans le but d'évaluer leur potentiel à la désexcitation. Seule une structure proposée récemment [22] permet d'avoir un rapport de saillance $s$ inférieur à 1 et offre donc un excellent compromis facteur de puissance / plage de fonctionnement à puissance constante. Remarquons que la composante démagnétisante de la force magnétomotrice ne doit pas désaimanter les aimants et que ce point doit être considéré attentivement lors de la conception du rotor. En outre, on peut constater que la réduction du flux d'entrefer à haute vitesse conduit à une diminution des pertes fer lorsque la vitesse augmente. Si l'on considère l'accroissement des pertes mécaniques et aérodynamiques avec la vitesse, le rendement reste, en pratique, sensiblement constant dans toute la plage à puissance constante. C'est ce que montrent notamment les caractéristiques des moteurs de la série LV de GEC-Alsthom Parvex [8, 18].

Au delà de cette étude, il reste de nombreux problèmes à étudier comme l'influence de la saturation magnétique que l'on pourrait mettre à profit, l'optimisation globale des paramètres dimensionnels de la machine pour rechercher un bon compromis entre ses performances (couple/pertes Joule...) et la plage de fonctionnement à puissance constante. Enfin, le risque de désaimantation des aimants doit être soigneusement analysé d'autant plus que l'évolution de leurs caractéristiques avec la température est importante. Dans le cas des néodyme-fer-bore, la valeur du champ limite de désaimantation diminue lorsque la température augmente.

\section{Bibliographie}

[1] Labraga M., Davat B. et Lajoie-Mazenc M., "Un servomoteur à aimants permanents ferrites pour entraînement direct" (R.G.E. 7 1982) pp. 38-42.

[2] Jahns T.M., Kliman G.B. et Neumann T.W., "Interior Permanent Magnet Synchronous Motors For Adjustable-Speed Drives" (IEEE IAS Conf. 1985) pp. 814-823.

[3] Jahns T.M., Flux-Weakening Regime Operation of an Interior Permanent-Magnet Synchronous Motor Drive, IEEE Trans. Ind. Appl. IA-23 (1987) 681-689.

[4] Sebastian T. et Slemon G.R., Operating Limits of Inverter-Driven Permanent Magnet Motor Drives, IEEE Trans. Ind. Appl. 23 (1987) 327-333.

[5] Labraga M., Davat B. et Lajoie-Mazenc M., "Conception d'un servomoteur à aimants permanents ferrites à entraînement direct", 5è Colloque sur les moteurs pas à pas, (Nancy, juin 1988) pp. 125134.

[6] Fratta A., Vagati A. et Villata F., AC Spindle Drives : a Unified Approach to the Field Weakening Behaviour (Motorcon, proc. 1988) pp. 44-56.

[7] Schifferl R.F. et Lipo T.A., Power Capability of Salient Pole Permanent Magnet Synchronous Motors in Variable Speed Drive Applications, IEEE Trans. Ind. Appl. 26, $\mathbf{N}^{\circ} 1$ (1990) 115-123. 
[8] Urgell J.J. et Régis A., "De nouvelles performances du moteur de broche grâce au contrôle du flux magnétique (G.F.C.)", J. SEE aimants permanents (Grenoble, S6/4 juin 1990) pp. 1-6.

[9] Multon B. et Glaize C., "Size Power Ratio Optimization for the Converters of Switched Reluctance Motors" (IMACS'TC1, Sept. 1990) pp 325-331.

[10] Morimoto S., Takeda Y., Hirasa T. et Taniguchi K., Expansion of Operating Limits for Permanent Magnet Motor by Current Vector Control Considering Inverter Capacity", IEEE Trans. Ind. Appl. 26 (1990) 866-871.

[11] Weh H., Mosebach h., Niemann W. et Tareilus A., "Fied Control in Synchronous Machines with Permanent Magnet Excitation in Flux Concentration Mode", (I.C.E.M., Boston, Vol. 1 1990) pp. 143-148.

[12] Fratta A., Vagati A. et Villata F., "Design Criteria of an I.P.M. Machine Suitable for Field Weakening", (I.C.E.M., Boston, 1990) pp. 1059-1065.

[13] Mühlegger W., Teppan W, et Rentmeister M., "Working Conditions of a Permanent Excited Synchronous Motor in Flux-Weakening Mode", (I.C.E.M., Boston, Vol. III 1990) pp. 1-4.

[14] Fratta A., Vagati A. et Villata F., "Permanent Magnet Assisted Synchronous Reluctance Drives for Constant Power Applications, Comparative Analysis of Control Requirements" (PCIM Conf., April 1992) pp. 187-195.

[15] Fratta A., Vagati A. et Villata F., "Extending the Voltage Saturated Performance of a DC Brushless Drive", (EPE, Firenze, Vol. 4, Sept. 91) pp. 134-138.

[16] Fratta A., Vagati A. et Villata F., "Permanent Magnet Assisted Synchronous Reluctance Drives for Constant Power Applications, Drive Power Limits" (PCIM Conf., proc. April 1992) pp. 196203.

[17] Hippner M. et Harley R.G., "Looking for an Optimal Rotor for High Speed Permanent Magnet Synchronous machine" IEEE IAS Conf. (Houston, Vol.1 1992) pp. 265-270.

[18] Maestre J.F., "Construction des moteurs synchrones à aimants" Journées électrotech. du club EEA, (Belfort, mars 1993) p. 9.

[19] Laporte B., Ibtouene R. et Chabane M. , "Optimisation d'une machine synchrone autopilotée à aimants insérés", Rev. Gen. Elec. 7/93 (1993) 25-31.

[20] Teixeira J.C., Chillet C., Yonnet J.P., "Structure Comparison of Buried Permanent Magnet Synchronous Motors for Flux Weakening Operation", 6th Conf. on E.M.D. (Oxford, Sept. 1993) pp. $365-370$.

[21] Soong W.L. et Miller T.J.E., "Practical Field-Weakening Performance of the Five Classes of Brushless Synchronous AC Motor Drive", EPE Brighton, Vol. 5, (13-16 September 1993) pp. 303-310.

[22] Xu L., Ye L. et El-Entably A., "A New Design Concept of Permanent Magnet Machine for Flux Weakening Operation" IEEE IAS Meeting, Vol.1 (Toronto, Oct. 1993) pp. 3-8.

[23] Yin H., Lin J.M. et Jin Z., "Comparison on Characteristics of PMSMs with Different Parameters for Flux-Weakening Operation" Conf. I.C.E.M., Vol.4 (Paris, Sept. 1994) pp. 1-5.

[24] Binns K.J. et Shimmin D.W., "Design Considerations for a Vector Controlled Permanent Magnet Motor with Wide Speed Range", Conf. I.C.E.M., Vol.3 (Paris, Sept. 1994) pp. 220-224.

[25] Morimoto S., Sanada M. et Takeda Y., Wide-Speed Operation of Interior Permanent Magnet Synchronous Motors with High Performance Current Regulator, IEEE Trans. Ind. Appl. 30 (1994) $920-926$.

[26] Llibre J.F. et Matt D., "Vernier Reluctance Magnet Machine for Electric Vehicle", Conf. I.C.E.M., Vol.1 (Paris, Sept. 1994) pp. 251-256.

[27] Liao Y. et Lipo T.A., "A New Doubly Salient Permanent Magnet Motor for Adjustable Speed Drives", Electric Machines and Power Systems, 22 (1994) pp. 259-270.

[28] Song W.L., Staton D.A. et Miller T.J.E., "Design of a New Axially-Laminated Interior Permanent Magnet Motor", I.E.E.E. IAS Meeting, Vol.1 (Toronto, Oct. 1993) pp. 27-36. 
[29] Zhang Z., Profumo F. et Tenconi A., "Axial Flux Interior PM Synchronous Motors for Electric Vehicle Drives", SPEEDAM, (Taoarmina, Italy, 8-10 June 1994) pp. 323-328.

[30] Chalmers B.J., "Influence of Saturation in Brushless Permanent Magnet Motor Drives", Correspondence in IEE Proc.-B, Vol.139 (January 1992) pp. 51-52. 